\title{
Surgery and quantum physics
}

\section{Part II: Light carries the signature of space-time}

\author{
Martin Riegler
}

(C) Springer-Verlag GmbH Austria, part of Springer Nature 2017

Dear reader,

This issue of European Surgery presents novel data on endocrine surgery, esophago-gastrointestinal surgery, colorectal surgery, hepato-bilio-pancreatico-digestive disease management, and treatment of infectious disorders of the skin. Again, it is important to point out that surgery becomes art if it adequately searches for a thorough understanding of the causes underlying the diseases, and if surgery includes this understanding into the orchestration of disease management. As such, each one of you, dear readers, has the opportunity to be more than merely a mechanical cutter of tissues and a rapid tissue-suturing device. If you open your mind to a deeper understanding of the diseases you have to treat, manage, and "heal," you may be allowed to introduce yourself into the fascinating and highly motivating world of the pathophysiology, anatomy, and histopathology of the human myth: being.

Physics represents a special form with which to tell the myth of being. Other forms of telling the myth of human being include logic, history, biology, medicine, architecture, art, astronomy, computer sciences, ethics etc. They all originate from the basic will and desire of man to understand his being.

In keeping with the recent issue of European Surgery, we aimed to gain a quantum physics-based understanding of life, and how life differs from dead matter. Let us briefly summarize the recent journey and see where we have reached.

Space, time, motion, change, and interactions are the basic tools for our perceptions, and our reasoning puts them together in the form of our "world,"

\section{Doz. Dr. M. Riegler $(\bowtie)$}

Reflux Medical Mariannengassse, 10/9, 1090 Wien, Austria martin.riegler@refluxmedical.com our "being." Special relativity aims to model the macroworld and teaches that energy equals mass, that light is the universal constant speed and form of electromagnetic energy.

As a consequence of the fact that there can be no faster speed for interaction and transmission of information than the speed of light, the following notion arises: compared with a system at continuous speed or at rest, moving clocks go slower, i. e., time passes slower if you move (dilatation of time), and moving structures get shorter (i. e., shortening of length). Thus, space and time fuse to form space-time: The movement of an object is compared to the movement of the hand of the watch. And this is how our world works without the inclusion of gravity.

General relativity is highly fascinating and examines the role, the effect, and the influence of gravitation on our world (Fig. 1). Gravitation is the geometry of space-time, there is no space without time and there is no time without space. Masses are energies that form the geometry of space-time. As such, masses define the curving of the geometry of space-time. Masses simply follow the geometry of space-time, which speeds them up. Gravitation is the geometry of space-time and defines the way (velocity, direction) objects move, speed up, and turn. Therefore, gravitation equals the geometry of space-time. Objects (masses) define how space-time curves and how space-time curving defines how objects move and how fast they "fall" (i. e., their velocity). Therefore, space-time and objects (masses) are different representations of the same energy: gravitation. In addition, it is important to understand that space-time comes in the form of a field, the gravitational field, which continuously moves, shakes, extends, contracts, and waves. Thus, gravitational waves alter the position of planets and galaxies, even the geometry of our earth (see LIGO project 2017 


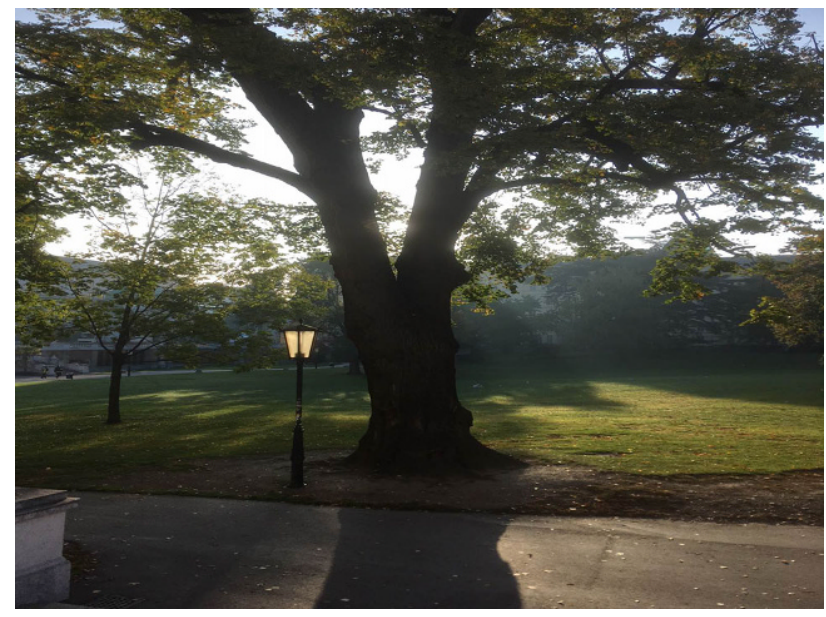

Fig. 1 The image mirrors the idea of the author that light carries the signature of space-time, as outlined in the text. The geometry of the space presented in the image is part of the same space-time that we share with distant galaxies in our universe and the space-time that zooms down to the mini black holes of the quantum world, as described in the text. Image obtained in September 2017 in the royal garden of Vienna

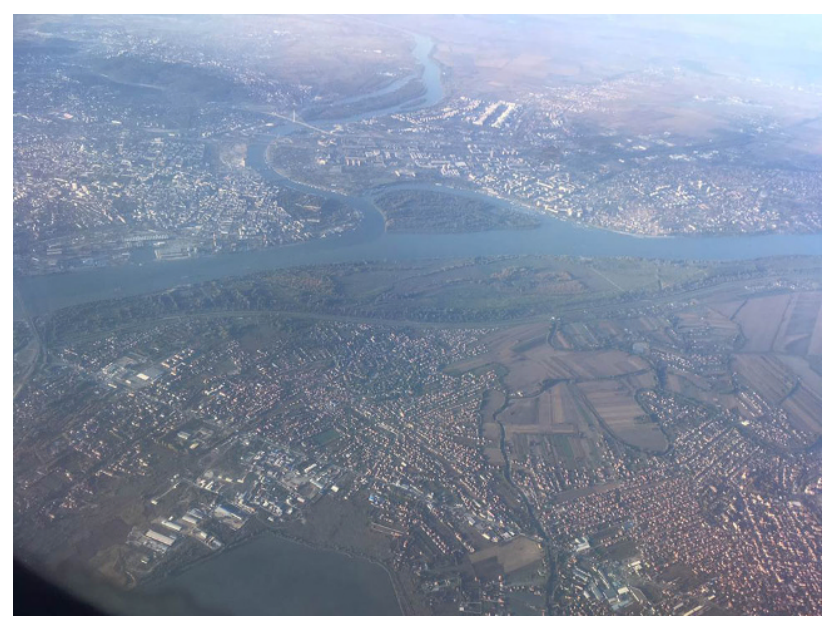

Fig. 2 The image shows the confluence of the rivers Danube (Ister) and Sava in Belgrade, depicting the idea of the author: change, movement, flow, interaction are the basis for all our perceptions. Image obtained during a flight to Belgrade, October 2017

Nobel Prize winner). In line with this understanding, light is deviated by the gravitational field and this explains the phenomenon of gravitation lenses, which mirror multiple images of a single galaxy, as seen by the Hubble telescope. Furthermore, the light coming from a distant star is deviated by the gravitational field of our sun, before reaching the lenses of our telescopes here on earth. Furthermore, clocks go faster when placed more distant to a mass when compared with the surface of a mass. All these issues are to be taken into account for the adequate operation of our World-Wide-Web-based satellite Google Earth navigation system. Taken together, the fusion

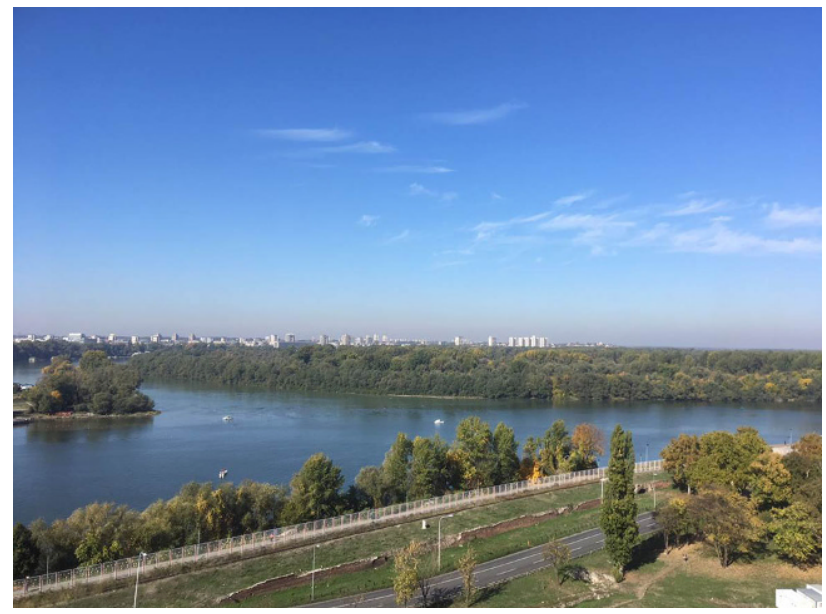

Fig. 3 The image shows a close-up view of the confluence of the rivers Danube (Ister) and Sava. Compared with Fig. 2, this image shows a more detailed zoomed-in view of the river confluence, depicting the idea that the world consists of energetic fields of quanta ranging from the micro- to the macroresolution of our perception

of special and general relativity teaches us that light carries the signature of space-time, the gravitational field (Fig. 2). As such, we can assume that masses represent the crystallization of the gravitational field, i. e., of space-time. Finally, we have to understand that space-time is continuously moving, turning and waving, and transmitting gravitational wave information going back to the origins of our universe. The so-called negative gravitation is responsible for the expansion of the universe and the positive gravitation orchestrates the interaction of masses to form galaxies, solar systems, planets, and YOU and us.

It remains to be asked: Why and how does this impact the life of a surgeon? Why should a surgeon care about relativity, space-time, and gravitation?

There is a simple answer to this important question: Disease happens in man, man happens in the universe, and disease and man and the universe follow the geometry of gravitation. Thus, you have to clean your operation field from adjacent organs, which "fall" into your operation field due to gravitation. As they follow the geometry of space-time in your operating theater, tissues, organs, fluids, secretions, and blood interfere with your vision. This is even true for your instruments and requires the adequate positioning of your patient during surgical procedures ( \pm robot, minimally invasive, endoscopic, NOTES, open). Hence, surgery consumes several activities to act against the manifestations of the gravitational field, i. e., space-time. And even when you schedule a case, you have to define the location and time with space-time data (the coordinates and the time point).

Now let us move to the microcosmos, where we search for a better understanding of the molecular pathology of normalcy, disease, inflammation, and 
cancer (Fig. 3). We have found that the microworld also comes in the form of fields.

The quantum world is made up of tiny quantum fields $\left(10^{-66} \mathrm{~cm}^{2}\right)$, which represent the quantum forms of energies (Planck quanta). When physicists interact with these quantum fields, they speak of particles and waves. These particles and waves are the basis for technologies used for the diagnosis and treatment of disease, including: computed tomography, magnetic resonance tomography, positron emission tomography, sonography, conventional radiology, computer-based translation and imaging of wave and particle information, as well as the use of radiofrequency and other radiation energies for the treatment of benign and oncologic diseases. One of the most fascinating phenomena in quantum physics is the observation that physicists cannot retrieve information beyond a particle size of $10^{-33} \mathrm{~cm}$. Here the energy applied during the measurement increases the mini space-time in such a manner that it produces a mini black hole. Thus, no additional information gets out of the system. It is the same as with so-called black holes in the macrocosmos. These are the highest concentrations of masses that curve space-time and thus increase gravitation. As a consequence, information (light) may enter but cannot escape the black hole. Therefore, we cannot get information about the content of black holes. A similar thing happens within the quantum world. And it is assumed that such mini black holes foster the development of genetic quantum effects for the development of genetic mutations and disease (inflammation, cancer). Future studies are awaited for a better quantum-based understanding of disease.

In the recent issue of European Surgery we reached the conclusion that life is quantum-based cytohumoral information orchestration (approximation, 5.1). However, an important thing is missing here. Life reproduces and reproduction is encoded within the genetic program. And the genetic program may change during the development of disease and cancer. Hence, we should extend our approximation and state:

Life is self-reproducing quantum genetics-based cytohumoral information orchestration (approximation, 5.1). By contrast, dead matter lacks quantum genetics.

In the next issue of European Surgery we may examine the role of quantum physics for our current understanding of disease (inflammation, cancer).

May you find relaxed space-time for your beloved families and friends during the upcoming seasonal festivities, which happen due to the fact that, during the course of a year, our beautiful earth falls around the sun, strictly following the space-time curving of the sun. Here we go: gravitation counts! Stay tuned and foster well-being!

Sincerely, Martin Riegler

Conflict of interest M. Riegler declares that he has no competing interests. 